\title{
NOVEL APPROACH TO MAGNETIC RESONANCE IMAGING OF EPILEPTIC DOGS - T2 RELAXOMETRY OF THE BRAIN WITH EMPHASISED HIPPOCAMPUS
}

\author{
Borbála A. LÖRINCZ ${ }^{1,2^{*}}$, Agustina ANSON ${ }^{2}$, Péter CSÉBI ${ }^{2}$, Gábor BAJZIK ${ }^{1}$, Gergely BIRÓ ${ }^{1}$, \\ Alexander TICHY ${ }^{2}$, Balázs B. LŐRINCZ ${ }^{3}$ and Rita GARAMVÖLGYI ${ }^{1}$ \\ ${ }^{1}$ Institute of Diagnostic Imaging and Radiation Oncology, Kaposvár University, \\ Guba S. u. 40, H-7400 Kaposvár, Hungary; ${ }^{2}$ Veterinary University Vienna, \\ Diagnostic Imaging, Veterinärplatz 1, A-1210 Vienna, Austria; ${ }^{3} \mathrm{Head}$ and Neck Center \\ of the University Medical Center Hamburg-Eppendorf, Hamburg, Germany
}

(Received 17 January 2017; accepted 4 April 2017)

Hippocampal sclerosis is the most common imaging finding of intractable human epilepsy, and it may play an important role in canine and feline epileptogenesis and seizure semiology, too. The magnetic resonance imaging (MRI) criteria of hippocampal sclerosis are T2 hyperintensity, shrinkage and loss of internal structure. The detection of these changes is often challenging by subjective visual assessment of qualitative magnetic resonance (MR) images. The recognition is more reliable with quantitative MR methods, such as T2 relaxometry. In the present prospective study including 31 dogs with idiopathic epilepsy and 15 control dogs showing no seizure activity, we compared the T2 relaxation times of different brain areas. Furthermore, we studied correlations between the hippocampal T2 values and age, gender and skull formation. We found higher hippocampal T2 values in the epileptic group than in the control; however, these findings were not statistically significant. No correlations were found with age, gender or skull formation. In the individual analysis six epileptic dogs presented higher hippocampal T2 relaxation times than the cut-off value. Two of these dogs were also evaluated as abnormal in the visual assessment. Individual analysis of hippocampal T2 relaxation times may be a helpful method to understand hippocampal involvement in canine epilepsy.

Key words: Canine, epilepsy, hippocampus, MRI, T2 relaxometry

The hippocampus, located in the medial temporal lobe of the forebrain belonging to the limbic system, plays an important role in human epilepsy. On one

*Corresponding author; E-mail: Borbala.Loerincz@vetmeduni.ac.at; Phone: 0043 (664) 60257-6460; Fax: 0043 (1) 25077-5790

This is an open-access article distributed under the terms of the Creative Commons Attribution License, which permits unrestricted use, distribution, and reproduction in any medium for non-commercial purposes, provided the original author and source are credited. 
hand, hippocampal sclerosis is the most common pathology underlying pharmacologically intractable cases (Bernasconi et al., 2000; Scott et al., 2003) of the so-called (mesial) temporal lobe epilepsy (TLE). In these particular cases, the hippocampus represents the seizure focus - i.e. the epileptogenic lesion (Rosanow and Lüders, 2001) - in the brain. On the other hand, there are also further forms of human epilepsy with a dual pathology, where hippocampal abnormalities are associated with extrahippocampal lesions generating the seizure focus (Okujava et al., 2002). In those cases, hippocampal changes are likely to be secondary to chronic seizuring. Whether TLE and the associated hippocampal sclerosis represent a discrete form of canine epilepsy is controversial. According to a study based on histological findings, TLE - if present - is not a common cause of medically intractable epilepsy in dogs (Buckmaster et al., 2002). However, hippocampal changes, especially hippocampal necrosis is a separate and quite common underlying cause of feline epilepsy resulting in seizures with orofacial involvement (Pákozdy et al., 2010, 2011).

Hippocampal changes in epilepsy are manifested as hippocampal atrophy or sclerosis. Histologically, there are evident neuronal loss, gliosis and secondary shrinking of the hippocampus (Grünewald et al., 1994; Bernasconi et al., 2000). With qualitative magnetic resonance imaging (MRI) hippocampal sclerosis can be detected subjectively. The MRI criteria of hippocampal sclerosis are volume loss, elevation of hippocampal signal intensities on T2-weighted (T2w) images indicating increased tissue-free water, and loss of internal structure (Okujava et al., 2002). There are some other pathologies causing high signal intensities on the water-sensitive MR sequences such as oedema, hyperaemia and inflammation, also detectable in the hippocampus as postictal features. In contrast to hippocampal sclerosis, those changes are reversible, without attendant volume loss (Mellema et al., 1999). Due to the subjective nature of the visual assessment of abnormal $\mathrm{T} 2 \mathrm{w}$ hippocampal signals, $\mathrm{T} 2$ relaxation times should be quantified to reduce subjectivity and increase sensitivity of the detection of hippocampal disease (Grünewald et al., 1994). In addition, visual detection is least reliable in case of mild changes or in bilateral abnormalities. Therefore, quantitative MR methods such as hippocampal volumetry and $\mathrm{T} 2$ relaxometry appear to be more helpful. Tissue hydration is quantified via $\mathrm{T} 2$ relaxometry, while hippocampal atrophy is quantified via hippocampal volumetry (Jack, 1996). In human medicine, T2 relaxometry is a quantitative magnetic resonance tool that can be used to increase the sensitivity of identifying hippocampal abnormalities above the level that visual assessment alone makes possible, so it can detect subtle changes that otherwise would not be considered to be hippocampal sclerosis (Scott et al., 2003). With this method, it is also possible to detect seizure-related alterations in $\mathrm{T} 2$ relaxation times of other cerebral structures, such as the temporal lobe white matter or the amygdala (Jack, 1996; Townsend et al., 2004). The T2 relaxation times - measured in milliseconds (ms) - are most likely MR equipment depend- 
ent. Hence, for the sake of reliable comparison, individual and statistical analysis settings, as well as the establishment of an equipment-related normal range is recommended (Grünewald et al., 1994; Okujava et al., 2002).

To our knowledge, no study about T2 relaxometry of the hippocampus in epileptic companion animals has yet been published in veterinary medicine, although the hippocampus may play an important role in canine and feline epileptogenesis and seizure semiology, in similar way as in human patients (Kuwabara et al., 2010; Pákozdy et al., 2011). An experimental rat model of hippocampal sclerosis provided the same results as seen in human studies (Parekh et al., 2010), but due to its experimental nature, it is not quite as conclusive in the veterinary practice.

A multi-observer study comparing hippocampi of epileptic and healthy cats relying only on visual assessment, showed moderate to good interobserver agreement, confirming the subjectivity and the unreliability of that method, too (Classen et al., 2016). There are several works studying hippocampal changes particularly using quantitative MR methods, such as hippocampal volumetry in epileptic companion animals, supporting the importance and role of the hippocampal formation in feline (Fatzer et al., 2000; Brini et al., 2004; Schmied et al., 2008; Mizoguchi et al., 2014) and canine epilepsy (Vullo et al., 1996; Kuwabara et al., 2010; Milne et al., 2013). These studies described the qualitative MR findings of hippocampal abnormalities (sclerosis, atrophy and necrosis) in dogs and cats. Furthermore, some of them provided results about normal and altered hippocampal volumes in healthy and epileptic dogs, relying on volumetric measurements and asymmetry ratios.

The aims of the present study were (1) to set an equipment-related normal value (mean and standard deviation, SD) of the hippocampal T2 relaxation times in a non-epileptic control group, to describe its correlation with age, gender, breed and skull formation, (2) to evaluate statistical differences in the hippocampal T2 relaxation times between the control and epileptic dog groups, (3) to conduct an individual analysis of hippocampal values in the epileptic group, and (4) to detect possible differences in the $\mathrm{T} 2$ relaxation times of other cerebral structures, such as the grey and white matter of the temporal and frontal lobes.

\section{Materials and methods}

\section{Subjects}

Two groups of client-owned dogs were included in this prospective study. The owners were informed about the study and signed a declaration of consent prior to imaging. 
The epileptic group comprised 31 dogs. For the presumptive diagnosis of primary epilepsy, all dogs met the requirements of the tier I confidence level by De Risio, besides laboratory tests being performed on each dog (De Risio et al., 2015).

The control group included 15 dogs. These had not shown any seizure activity or brain-associated neurological signs in their entire life, and were presented for MR imaging for other reasons.

\section{Image acquisition}

All dogs underwent general anaesthesia using propofol premedication intravenously (Narcofol ${ }^{\circledR}$, CP-Pharma $\mathrm{GmbH}, 4-7 \mathrm{mg} / \mathrm{kg}$ body weight bolus injection). Following intubation, the narcosis was maintained using isoflurane-oxygen inhalation (Forene ${ }^{\circledR}$, AbbVie Deutschland GmbH \& Co, $1-5$ vol. \%, oxygen flow $2-3 \mathrm{l} / \mathrm{min})$.

The magnetic resonance (MR) examinations were performed using a $1.5 \mathrm{~T}$ MR scanner (Siemens Magnetom Avanto, Siemens, Erlangen, Germany) in ventral recumbency. The same MR protocol was used consistently in each case, covering the entire brain : T2w images in transversal [time of echo/time of repetition $(\mathrm{TE} / \mathrm{TR})=105 / 2900 \mathrm{~ms}$, slice thickness $(\mathrm{SL})=3 \mathrm{~mm}]$, sagittal $(\mathrm{TE} / \mathrm{TR}=105 /$ $2900 \mathrm{~ms}, \mathrm{SL}=3 \mathrm{~mm}$ ), and paradorsal planes (TE/TR $=105 / 4520 \mathrm{~ms}, \mathrm{SL}=3 \mathrm{~mm}$ ), fluid-attenuated inversion recovery (FLAIR) images in transversal plane (TE/TR $=$ $113 / 8500 \mathrm{~ms}, \mathrm{SL}=3 \mathrm{~mm}$ ), time of flight $(\mathrm{ToF})$ angiographic images in transversal plane $(\mathrm{TE} / \mathrm{TR}=7.15 / 25 \mathrm{~ms}, \mathrm{SL}=1 \mathrm{~mm})$, T1-weighted $(\mathrm{T} 1 \mathrm{w})$ thin sliced magnetisation prepared rapid gradient-echo (MP-RAGE) images in sagittal plane $(\mathrm{TE} / \mathrm{TR}=4.24 / 913 \mathrm{~ms}, \mathrm{SL}=0.9 \mathrm{~mm})$ and reconstructions in transversal and paradorsal planes. The field of view (FoV) used for all measurements corresponded $224 \times 320 \mathrm{~mm}$. The paradorsal planes $(\mathrm{T} 2 \mathrm{w}, \mathrm{T} 1 \mathrm{w}$ MP-RAGE and T2 relaxometry) were oriented perpendicular to the long axis of the hippocampus with planning and placing the paradorsal slice on the sagittal plane - as described and recommended previously (Hasboun et al., 1996; Milne et al., 2013).

T2 relaxometry studies were performed during the same anaesthesia and positioning, as well as using the same MR equipment with spin echo (SE) sequence (FA: 180 degree; FoV: $230 \mathrm{~mm} \times 201 \mathrm{~mm}$; acquisition matrix: $512 \times$ 448; SL: $5.0 \mathrm{~mm}$; TR: $2450 \mathrm{~ms}$; averages: 1). Each slice was scanned with $16 \mathrm{TE}$ values ranging from $22 \mathrm{~ms}$ to $352 \mathrm{~ms}$ with equal intervals of $22 \mathrm{~ms}$. Eight slices were scanned for each animal with a slice spacing of $10 \mathrm{~mm}$ (slice gap: $5 \mathrm{~mm}$ ). T2 maps were calculated from the multi-echo sequence using the Siemens Syngo Dynamic Analysis package. Largest possible regions of interest (RoI) were traced manually on the T1 PD image series (Fig. 1), which had also been derived from the multi-echo sequence by the software. Boundaries to cerebrospinal fluid (CSF) were avoided to minimise partial volume artefacts. RoIs were then transferred and measured on the T2 maps (Fig. 2) using ITK-SNAP 3.4.0 (Yushkevich et al., 2006). Values of the hippocampus as well as those of the white and 
grey matter of the temporal and frontal lobes were measured separately in both hemispheres. Figs 1 and 2 show the requirements of using T1 PD images for tracing the RoIs on account of the poor contrast on the T2 maps. For better visualisation, find the colour key in Fig. 3.

MR images of the epileptic dogs were reviewed independently by two observers (B.A.L. and A.A.) blinded to the clinical findings.
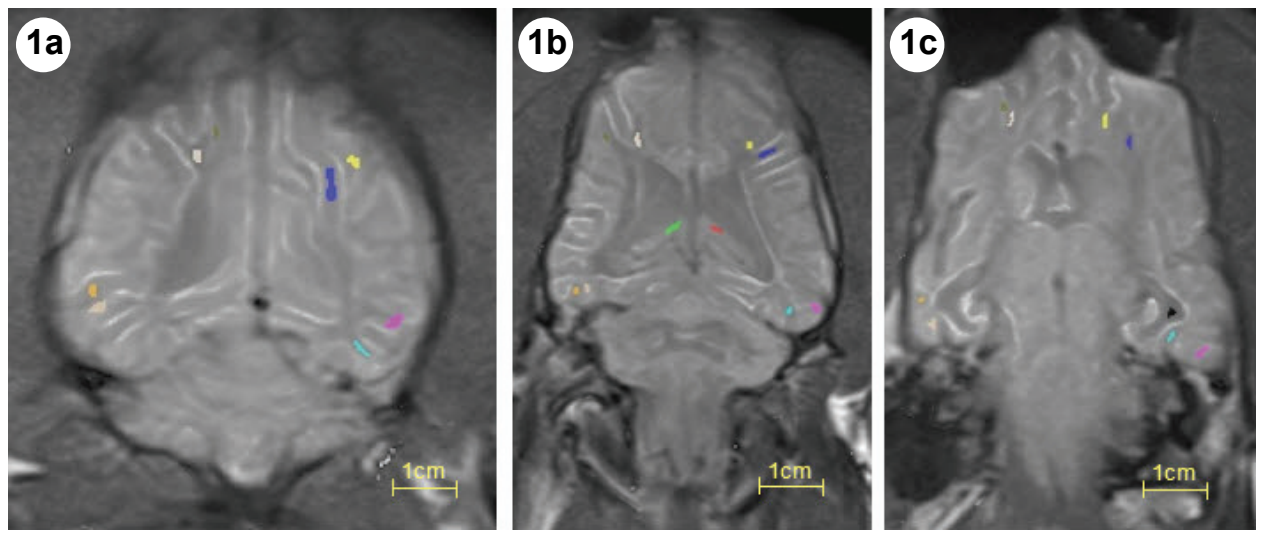

Fig. 1. Manually traced free hand regions of interest (RoIs) in T1 paradorsal (PD) images - dorsal slice at the level just dorsal to the adhesio interthalamica (1/a), middle slice at the level of the mesencephalon $(1 / b)$, and ventral slice at the level of the brainstem (1/c). Left/right label descriptions, respectively: hippocampus - red/green; frontal lobe grey matter - blue/brown; frontal lobe white matter - yellow/grey; temporal lobe grey matter - pink/beige; temporal lobe white matter - turquoise/orange

\section{Statistical analysis}

Statistical analysis was performed using IBM SPSS Version 22 (IBM Corp., Armonk, NY). Median, mean and SD were calculated for each group and for each anatomical area separately in both hemispheres (hippocampus, frontal and temporal white and grey matter). Ratios between hippocampal values and temporal white and grey matter values were explored separately in both groups. A two-step cluster analysis for groups, genders and skull formation (brachycephalic dogs) was also performed. For individual analysis, data were ztransformed. Values lying outside the range of two SDs were suggestive of hippocampal alterations. Kolmogorov-Smirnov test was applied to test the assumption of normal distribution. Due to the lack of the latter, data were analysed using nonparametric tests. Mann-Whitney tests were used to analyse differences between groups and between genders, while Wilcoxon tests were used to compare values between the left and the right side, as well as among the anatomical regions within each side. The correlation between hippocampal values and age was assessed using Spearman's correlation coefficient. 

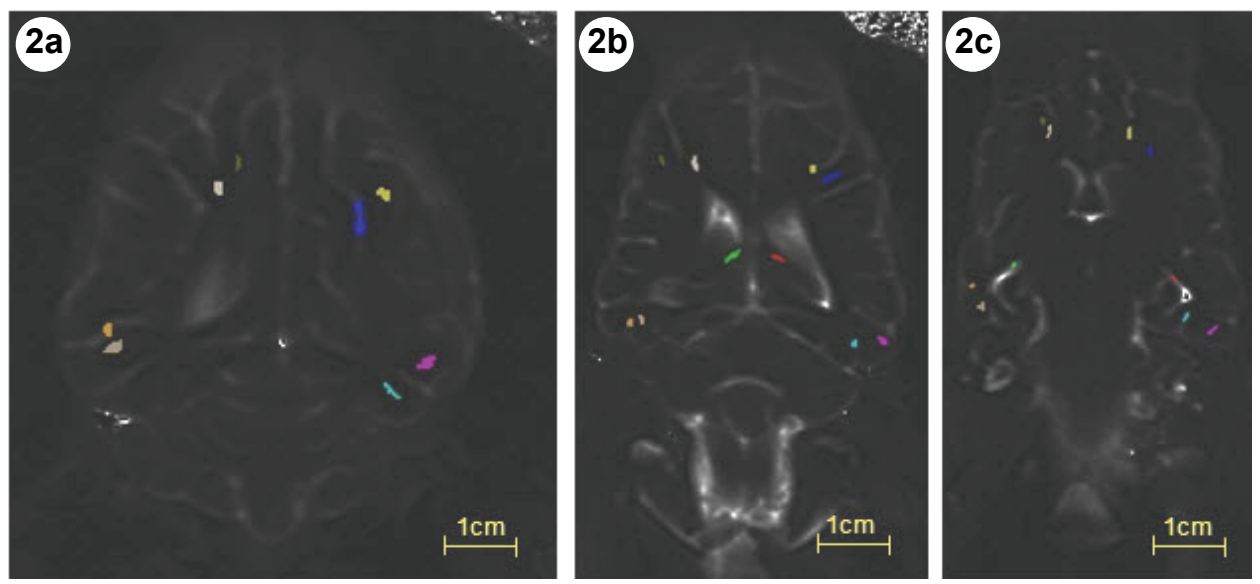

Fig. 2. Transferred regions of interest (RoI) in the T2 maps corresponding to the T1 PD images dorsal slice at the level just dorsal to the adhesio interthalamica (2/a), middle slice at the level of the mesencephalon $(2 / b)$ and ventral slice at the level of the brainstem $(2 / \mathrm{c})$. Left/right label descriptions: see legend to Fig. 1

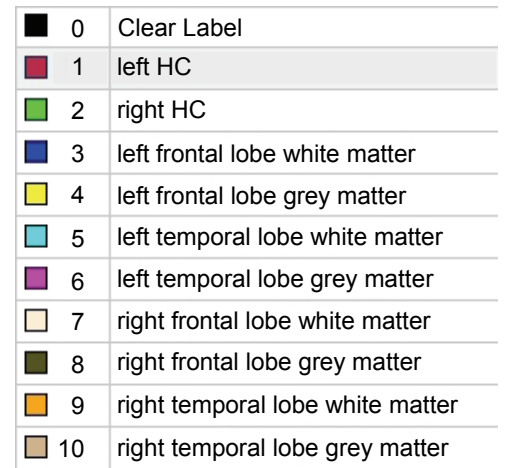

Fig. 3. Colour key of the manually placed and transferred regions of interest

\section{Results}

In the epileptic group there were 20 males and 11 females (a total of 31 dogs), aged between 0.3 and 10.5 years $(4.3 \pm 2.37$ years) and weighing between 1.8 and $72 \mathrm{~kg}(23 \pm 16 \mathrm{~kg})$. The dogs represented the following breeds: mongrel $(\mathrm{n}=6)$, German Shepherd, French Bulldog and Hungarian Vizsla $(\mathrm{n}=3)$, Golden Retriever and Bolognese $(\mathrm{n}=2)$, as well as one each of the following breeds: Miniature Schnauzer, Sarplaninac, Cocker Spaniel, Pit Bull Terrier, Moscow Watchdog, Husky, Jack Russell Terrier, Beagle, Dachshund, American Bulldog, Australian Shepherd and Rhodesian Ridgeback. The dogs were referred to MRI by 19 veterinary practitioners with a suspected diagnosis of idiopathic epilepsy, 
to exclude structural brain lesions. All dogs underwent physical and neurological examination by the practitioner, and showed interictally no neurological signs. Serum biochemistry were performed in 15 cases, abdominal ultrasonography in 3 cases, EEG in 2 cases, and PET-CT in a single case. All findings were normal, apart from one dog with mildly elevated liver enzymes. This particular dog had been treated with phenobarbital for several years. The neurological ictal signs were generalised seizures $(\mathrm{n}=11)$, focal seizures with secondary generalisation $(n=4)$, or focal seizures $(n=8)$. In 8 cases, no additional information was available regarding their seizure semiology.

The control group involved 11 males and 4 females (a total of 15 dogs), aged between 2 and 8 years ( $4.4 \pm 7$ years) and weighing between 4 and $32 \mathrm{~kg}$ $(15.7 \pm 9.7 \mathrm{~kg})$. These dogs represented the following breeds: mongrel $(\mathrm{n}=8)$, Boxer $(n=2)$, Dachshund $(n=2)$, Miniature Pinscher $(n=1)$, Spitz $(n=1)$, and Welsh Corgi $(\mathrm{n}=1)$.

Mild ventriculomegaly was present in two dogs in the control group $(13.3 \%)$ and in 5 dogs in the epileptic group (16\%) without any evidence of elevated intracranial pressure (Laubner et al., 2015). The findings were interpreted as breed-related normal findings (brachycephalic dogs) (Ryan et al., 2013). Both observers found all hippocampal formations with normal signal intensity in all sequences and there was $100 \%$ interobserver agreement regarding no other structural abnormalities recognised in the brain by visual assessment in both groups of dogs.

One of the observers evaluated subjectively by visual assessment the hippocampal volume as altered in the epileptic group: in four dogs as asymmetry and in another four dogs as suspected mild bilateral shrinkage. This resulted in a $74 \%$ interobserver agreement with subjective visual assessment regarding the hippocampal volume.

T2 relaxation times of the different brain areas and groups are shown in Table 1. Our data showed that the mean hippocampal T2 relaxation times were higher in the epileptic group than in the control group (Fig. 4), but the differences were not statistically significant. Further, no statistically significant differences could be found in the T2 relaxation times of the frontal and temporal lobe white and grey matter between the groups. There were no significant differences between the hippocampus and the temporal lobe white and grey matter in these groups. However, we found that the difference between the frontal lobe grey matter and the hippocampus was almost statistically significant in the epileptic group ( $\mathrm{P}=0.063$ on both sides), while the same comparison was far from being significant in the control group $(\mathrm{P}=0.570$ on the left and $\mathrm{P}=0.173$ on the right side). There was no correlation among the hippocampal T2 values and age, gender or breed in either group. According to the results of the cluster analysis, skull formation - i.e. brachycephalic vs. non-brachycephalic dogs - did not have any effect on hippocampal T2 relaxation times in either group. 
Table 1

$\mathrm{T} 2$ relaxation times of each brain area in the control and epileptic groups

\begin{tabular}{lcc}
\hline \multirow{2}{*}{ Brain area } & \multicolumn{2}{c}{ T2 relaxation times (ms) (mean/median \pm SD) } \\
\cline { 2 - 3 } & Control group & Epileptic group \\
\hline Left hippocampus & $127.9 / 117.4 \pm 44.4$ & $144.2 / 113.4 \pm 61.9$ \\
Right hippocampus & $137.5 / 122.9 \pm 38.4$ & $141.6 / 119.0 \pm 55.2$ \\
Left frontal lobe white matter & $120.9 / 113.8 \pm 17.8$ & $115.4 / 109.5 \pm 19.0$ \\
Right frontal lobe white matter & $124.5 / 113.7 \pm 28.7$ & $117.5 / 110.2 \pm 27.8$ \\
Left frontal lobe grey matter & $124.3 / 114.4 \pm 31.5$ & $119.0 / 105.8 \pm 43.4$ \\
Right frontal lobe grey matter & $122.5 / 121.2 \pm 22.8$ & $117.2 / 106,3 \pm 29.4$ \\
Left temporal lobe white matter & $111.8 / 107.0 \pm 19.3$ & $130.2 / 126.7 \pm 33.6$ \\
Right temporal lobe white matter & $115.0 / 105.2 \pm 23.1$ & $142.5 / 119.6 \pm 58.8$ \\
Left temporal lobe grey matter & $110.7 / 104.2 \pm 26.3$ & $107.1 / 99.4 \pm 23.9$ \\
Right temporal lobe grey matter & $106.3 / 97.2 \pm 26.6$ & $112.1 / 101.0 \pm 29.9$ \\
\hline
\end{tabular}

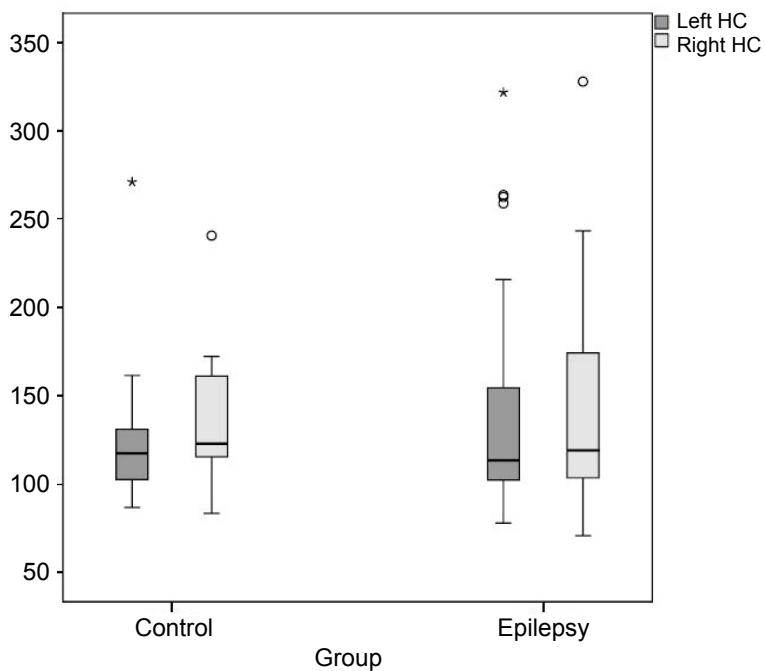

Fig. 4. Boxplot representing the means and standard deviations (SDs) of the left and right hippocampal T2 relaxation times (ms) in the epileptic and control groups

In the individual analysis, values higher than two SDs above the mean of normal controls were considered abnormal (Bernasconi et al., 2000). In the epileptic group six dogs showed elevated hippocampal T2 relaxation values: two of them bilaterally (the left/right hippocampal values: $321.8 / 327.9 \mathrm{~ms}, 262.6 /$ $243.4 \mathrm{~ms})$, three of them on the left $(215.8 \mathrm{~ms}, 263.6 \mathrm{~ms}$ and $258.9 \mathrm{~ms})$ and one of them on the right side $(218.4 \mathrm{~ms})$. Two dogs were presented with generalised seizures (one of them showed bilaterally elevated $\mathrm{T} 2$ relaxation times), two with 
focal seizures, one with focal seizures and secondary generalisation (showing bilaterally elevated $\mathrm{T} 2$ relaxation times), and one was presented with unknown seizure semiology. One of the observers found two of these six dogs showing abnormal findings in their visual assessment.

\section{Discussion}

Due to the lack of reliable EEG data, the diagnosis and classification of canine epilepsy is associated with considerably higher uncertainty than its human counterpart. Whether the (mesial) TLE represents an autotelic epileptic syndrome in dogs is still unknown. The alteration of the hippocampal formation i.e. hippocampal atrophy or sclerosis - secondary to epileptic activity has already been published in a rat (Parekh et al., 2010) and in two canine models (Hasegawa et al., 2002; Hasegawa et al., 2003). No study has been published about T2 relaxometry of the hippocampus in dogs.

In a volumetric study involving 58 epileptic dogs, the authors found a significantly greater hippocampal volumetric asymmetry ratio in epileptic dogs than in the control group, which is suitable for detecting unilateral, but not bilateral changes (Kuwabara et al., 2010). According to human studies, hippocampal relaxometry is more sensitive than volumetry to determine hippocampal atrophy, and it can also detect bilateral abnormalities (Grünewald et al., 1994; Bernasconi et al., 2000; Okujava et al., 2002; Scott et al., 2003).

We did not find any correlation among the hippocampal T2-relaxation times and age, gender, breed or skull formation. These findings are in line with human results showing no correlation between the hippocampal T2 relaxation times and age (Bernasconi et al., 2000; Scott et al., 2003). We did not find any relevant human data regarding gender or race. Due to the lack of data about seizure semiology in the epileptic group, we were not able to study whether seizure semiology is similarly indifferent to hippocampal relaxation times as it has been shown in human studies (Grünewald et al., 1994; Okujava et al., 2002).

There were no significant differences in the imaging of the brain structures apart from those in the hippocampus (such as e.g. the temporal lobe white matter), despite the fact that such differences have been found in human medicine (Townsend et al., 2004). Our results from comparing the values of the frontal lobe grey matter and the hippocampus were close to statistical significance in the epileptic group, while the respective results in the control group were far from being significant. However, a larger group size or a more detailed seizure classification may provide other findings. The changes between the temporal lobe grey matter and the hippocampus did not show significant differences. One possible reason for that is the difficulty of making a distinction between the grey and the white matter of the temporal lobe due to moderate spatial resolution of the im- 
ages. A possible contamination by placing the RoIs may also have modified our results, presenting more likely in smaller dogs due to the relatively thicker slices. The almost similar values of the temporal lobe white and grey matter make this hypothesis likely.

For the further assessment, it was necessary for us to define a mean hippocampal T2 relaxation time value in the control group. In human studies, there are several different mean hippocampal values, and therefore different cut-off values as well. Our results $(127.9 \pm 44.4 \mathrm{~ms}$ for the left and $137.5 \pm 38.4 \mathrm{~ms}$ for the right hippocampus) are similar to those of a human study with $133 \pm 4$ ms normal value (Okujava et al., 2002), but different from some other studies with normal values of $80 \pm 1.4 \mathrm{~ms}$ (Bernasconi et al., 2000) or a cut-off value of $116 \mathrm{~ms}$ (Grünewald et al., 1994). It is important to determine the machine-related normal range for further assessments. There are obviously larger SDs in our study compared to human results (ten times higher on average). Due to the diversity of canine skull formation it is challenging to place the free hand RoIs always accurately in the same brain area. In addition, the moderate spatial resolution could cause some contamination leading to more dispersed data. Further studies should be made with thinner slices and without interslice gap for better defined data collection.

In the individual analysis, we found 6 dogs showing higher hippocampal values than our cut-off value, two with bilateral and four with unilateral changes. These findings may match hippocampal sclerosis or atrophy. In the subjective visual assessment, two of these six dogs were suspected of having hippocampal changes. Further investigations - such as volumetric measurement and histopathology - would be necessary to prove hippocampal atrophy in these cases.

According to our data, visual assessment is quite unreliable due to its subjective nature. We found only a moderate interobserver agreement with regard to the suspected shrinkage of the hippocampus, without any apparent signal intensity changes in the qualitative MR images. In some of our cases, hippocampal changes were suspected in the visual assessment while showing normal T2 relaxation times, and vice versa. The results of a human study correspond to our findings, where T2 measurements confirmed the presence of probable hippocampal abnormalities that were detected by visual evaluation only in $63.6 \%$ of the cases. On the other hand, $36.4 \%$ of the visually suspicious changes were not confirmed by T2-relaxometry (Okujava et al., 2002). However, in human studies and in an experimental rat model as well, hippocampal T2 mapping provided evidence of hippocampal damage in the majority of TLE cases, without evidence of hippocampal atrophy on MRI (Bernasconi et al., 2000; Parekh et al., 2010). Moreover, T2-relaxometry makes it possible to detect mild, bilateral abnormalities in contrast to volumetric measurements (Grünewald et al., 1994). Visual assessment of qualitative MR images is the most subjective and inaccurate method, which may explain a certain disagreement within the relaxometric data. In our 
study, the different judgement of one of the observers according to the subjective hippocampal volume loss does indeed point out a possible bias.

$\mathrm{T} 2$ relaxometry is based on the ability of the technique to distinguish precisely between subtle unilateral or mild bilateral hippocampal changes and preeminently sclerosis. More obvious alterations, such as necrosis, inflammation or neoplasia are also visible on water-sensitive qualitative MR sequences (Jack, 1996; Pákozdy et al., 2011). Therefore, in case of obvious lesions on qualitative MR images, there is no need to use quantitative techniques to establish a radiological diagnosis (Jack, 1996).

Limitations of this study were the heterogeneous preimaging examinations as well as the missing video analysis of the seizures, and the lack of histologically proven hippocampal sclerosis. Furthermore, there may be certain limiting factors in the image acquisition, such as the proportionally too thick slices in smaller dogs, gapping and possible contamination by the placement the RoIs.

According to our results, we suggest that hippocampal sclerosis-related T2 relaxation time changes may be present in a certain group of epileptic dogs. This group of dogs may possibly have temporal lobe epilepsy with accompanying hippocampal sclerosis, presenting most likely as a subtype of canine epilepsy. Individual analysis seems to be a suitable method to prove hippocampal involvement in epileptic dogs, after setting an equipment-related normal range. Further investigations are needed with larger case numbers relying on more homogeneous preimaging examinations, as well as with measurements confirmed by histological and volumetric comparative data.

\section{Acknowledgements}

The authors thank András Szántó RT for his technical support, the referring veterinary practitioners, as well as Michael Maier for his English proofreading.

\section{References}

Bernasconi, A., Bernasconi, N., Caramanos, Z., Reutens, D. C., Andermann, F., Dubeau, F. Tampieri, D., Pike, B. G. and Arnold, D. L. (2000): T2 relaxometry can lateralize mesial temporal lobe epilepsy in patients with normal MRI. Neuroimage 12, 739-746.

Brini, E., Gandini, G., Crescio, I., Fatzer, R. and Casalone, C. (2004): Necrosis of hippocampus and piriform lobe: clinical and neuropathological findings in two Italian cats. J. Feline Med. Surg. 6, 377-381.

Buckmaster, P. S., Smith, M. O., Buckmaster, C. L., LeCouteur, R. A. and Dudek, F. E. (2002): Absence of temporal lobe epilepsy pathology in dogs with medically intractable epilepsy. J. Vet. Intern. Med. 16, 95-99.

Classen, A. C., Kneissl, S., Lang, J., Tichy, A. and Pakozdy, A. (2016): Magnetic resonance features of feline hippocampus in epileptic and non-epileptic cats: a blinded, retrospective and multi-observer study. BMC Vet. Res. 12, 165. 
De Risio, L., Bhatti, S., Muñana, K., Penderis, J., Stein, V., Tipold, A., Berendt, M., Farqhuar, R., Fischer, A., Long, S., Mandigers, P. J., Matiasek, K., Packer, R. M., Pakozdy, A., Patterson, N., Platt, S., Podell, M., Potschka, H., Battle, M. P., Rusbridge, C. and Volk, H. A. (2015): International epilepsy task force consensus proposal: diagnostic approach to epilepsy in dogs. BMC Vet. Res. 11, 148.

Fatzer, R., Gandini, G., Jaggy, A., Doherr, M. and Vandevelde, M. (2000): Necrosis of hippocampus and piriform lobe in 38 domestic cats with seizures: a retrospective study on clinical and pathologic findings. J. Vet. Intern. Med. 14, 100-104.

Grünewald, R. A., Jackson, G. D., Connelly, A. and Duncan, J. S. (1994): MR detection of hippocampal disease in epilepsy: factors influencing T2 relaxation time. Am. J. Neuroradiol. 15, 1149-1156.

Hasboun, D., Chantôme, M., Zouaoui, A., Sahel, M., Deladoeuille, M., Sourour, N., Duyme, M., Baulac, M., Marsault, C. and Dormont, D. (1996): MR determination of hippocampal volume: comparison of three methods. Am. J. Neuroradiol. 17, 1091-1098.

Hasegawa, D., Orima, H., Fujita, M., Hashizume, K. and Tanaka, T. (2002): Complex partial status epilepticus induced by a microinjection of kainic acid into unilateral amygdala in dogs and its brain damage. Brain Res. 955, 174-182.

Hasegawa, D., Orima, H., Fujita, M., Nakamura, S., Takahashi, K., Ohkubo, S., Igarashi, H. and Hashizume, K. (2003): Diffusion-weighted imaging in kainic acid-induced complex partial status epilepticus in dogs. Brain Res. 983, 115-127.

Jack, C. R. (1996): Hippocampal T2 relaxometry in epilepsy: past, present, and future. Am. J. Neuroradiol. 17, 1811-1814.

Kuwabara, T., Hasegawa, D., Kobayashi, M., Fujita, M. and Orima, H. (2010): Clinical magnetic resonance volumetry of the hippocampus in 58 epileptic dogs. Vet. Radiol. Ultrasound 51, 485-490.

Laubner, S., Ondreka, N., Failing, K., Kramer, M. and Schmidt, M. J. (2015): Magnetic resonance imaging signs of high intraventricular pressure - comparison of findings in dogs with clinically relevant internal hydrocephalus and asymptomatic dogs with ventriculomegaly. BMC Vet. Res. 11, 181.

Mellema, L. M., Koblik, P. D., Kortz, G. D., LeCouteur, R. A., Chechowitz, M. A. and Dickinson, P. J. (1999): Reversible magnetic resonance imaging abnormalities in dogs following seizures. Vet. Radiol. Ultrasound 40, 588-595.

Milne, M. E., Anderson, G. A., Chow, K. E., O’Brien, T. J., Moffat, B. A. and Long, S. N. (2013): Description of technique and lower reference limit for magnetic resonance imaging of hippocampal volumetry in dogs. Am. J. Vet. Res. 74, 224-231.

Mizoguchi, S., Hasegawa, D., Kuwabara, T., Hamamoto, Y., Ogawa, F., Fujiwara, A., Matsuki, N. and Fujita, M. (2014): Magnetic resonance volumetry of the hippocampus in familial spontaneous epileptic cats. J. Epilepsy Res. 108, 1940-1944.

Okujava, M., Schulz, R., Ebner, A. and Woermann, F. G. (2002): Measurement of temporal lobe T2 relaxation times using a routine diagnostic MR imaging protocol in epilepsy. J. Epilepsy Res. 48, 131-142.

Pákozdy, Á., Gruber, A., Kneissl, S., Leschnik, M., Halasz, P. and Thalhammer, J. G. (2011): Complex partial cluster seizures in cats with orofacial involvement. J. Feline Med. Surg. 13, 687-693.

Pákozdy, Á., Leschnik, M., Sarchahi, A. A., Tichy, A. G. and Thalhammer, J. G. (2010): Clinical comparison in primary versus secondary epilepsy in 125 cats. J. Feline Med. Surg. 12, 910-916.

Parekh, M. B., Carney, P. R., Sepulveda, H., Norman, W., King, M. and Mareci, T. H. (2010): Early MR diffusion and relaxation changes in the parahippocampal gyrus precede the onset of spontaneous seizures in an animal model of chronic limbic epilepsy. Exp. Neurol. 224, $258-270$.

Rosanov, F. and Lüders, H. (2001): Presurgical evaluation of epilepsy. Brain 124, 1683-1700. 
Ryan, C. T., Glass, E. N., Seiler, G., Zwingenberger, A. L. and Mai, W. (2014): Magnetic resonance imaging findings associated with lateral cerebral ventriculomegaly in English Bulldogs. Vet. Radiol. Ultrasound 55, 292-299.

Schmied, O., Scharf, G., Hilbe, M., Michal, U., Tomsa, K. and Steffen, F. (2008): Magnetic resonance imaging of feline hippocampal necrosis. Vet. Radiol. Ultrasound 49, 343-349.

Scott, R. C., Cross, J. H., Gadian, D. G., Jackson, G. D., Neville, B. G. R. and Connelly, A. (2003): Abnormalities in hippocampi remote from the seizure focus: a T2 relaxometry study. Brain 126, 1968-1974.

Townsend, T. N., Bernasconi, N., Pike, G. B. and Bernasconi, A. (2004): Quantitative analysis of temporal lobe white matter T2 relaxation time in temporal lobe pilepsy. Neuroimage 23, 318-324.

Vullo, T., Deo-Narine, V., Stallmeyer, M. J., Gomez, D. G. and Cahill, P. T. (1996): Quantitation of normal canine hippocampus formation volume: correlation of MRI with gross histology. J. Magn. Reson. Imaging 14, 657-662.

Yushkevich, P. A., Piven, J., Hazlett, H. C., Smith, R. G., Ho, S., Gee, J. C. and Gerig, G. (2006): User-guided 3D active contour segmentation of anatomical structures: significantly improved efficiency and reliability. Neuroimage 31, 1116-1128. 